\title{
Modulation of gene expression in endothelial cells in response to high LET nickel ion irradiation
}

\author{
MICHAËL BECK ${ }^{1,2}$, CHARLOTTE ROMBOUTS ${ }^{1,2}$, MARJAN MOREELS ${ }^{1}$, AN AERTS $^{1}$, \\ ROEL QUINTENS $^{1}$, KEVIN TABURY ${ }^{1}$, ARLETTE MICHAUX ${ }^{1}$, ANN JANSSEN ${ }^{1}$, \\ MIEKE NEEFS $^{1}$, ERIC ERNST ${ }^{3}$, BIRGER DIERIKS ${ }^{2,4}$, RYONFA LEE $^{5}$, WINNOK H. DE VOS ${ }^{2,4,6}$, \\ CHARLES LAMBERT $^{3}$, PATRICK VAN OOSTVELDT ${ }^{2,4}$ and SARAH BAATOUT ${ }^{1,2}$
}

\begin{abstract}
${ }^{1}$ Laboratory of Radiobiology, Institute for Environment, Health and Safety, Belgian Nuclear Research Centre (SCK-CEN), Mol; ${ }^{2}$ Department of Molecular Biotechnology, Ghent University, Ghent; ${ }^{3}$ Laboratory of Connective Tissues Biology, GIGA-Cancer, University of Liège, Liège; ${ }^{4}$ NB-Photonics, Ghent University, Ghent, Belgium; ${ }^{5}$ Biophysics Department, GSI Helmholtzzentrum für Schwerionenforschung, Darmstadt, Germany; ${ }^{6}$ Laboratory of Cell Biology and Histology,

Department of Veterinary Sciences, Antwerp University, Antwerp, Belgium
\end{abstract}

Received February 9, 2014; Accepted May 6, 2014

DOI: $10.3892 /$ ijmm.2014.1893

\begin{abstract}
Ionizing radiation can elicit harmful effects on the cardiovascular system at high doses. Endothelial cells are critical targets in radiation-induced cardiovascular damage. Astronauts performing a long-term deep space mission are exposed to consistently higher fluences of ionizing radiation that may accumulate to reach high effective doses. In addition, cosmic radiation contains high linear energy transfer (LET) radiation that is known to produce high values of relative biological effectiveness (RBE). The aim of this study was to broaden the understanding of the molecular response to high LET radiation by investigating the changes in gene expression in endothelial cells. For this purpose, a human endothelial cell line (EA.hy926) was irradiated with accelerated nickel ions (Ni) (LET, $183 \mathrm{keV} /$ $\mu \mathrm{m})$ at doses of 0.5, 2 and 5 Gy. DNA damage was measured 2 and $24 \mathrm{~h}$ following irradiation by $\gamma-\mathrm{H} 2 \mathrm{AX}$ foci detection by fluorescence microscopy and gene expression changes were measured by microarrays at 8 and $24 \mathrm{~h}$ following irradiation. We found that exposure to accelerated nickel particles induced a persistent DNA damage response up to $24 \mathrm{~h}$ after treatment. This was accompanied by a downregulation in the expression of a multitude of genes involved in the regulation of the cell cycle and an upregulation in the expression of genes involved in cell cycle checkpoints. In addition, genes involved in DNA damage response, oxidative stress, apoptosis and cell-cell signaling
\end{abstract}

Correspondence to: Professor Sarah Baatout, Laboratory of Radiobiology, Molecular and Cellular Biology Expert Group, Institute for Environment, Health and Safety, Belgian Nuclear Research Centre (SCK-CEN), Boeretang 200, $2400 \mathrm{Mol}$, Belgium

E-mail: sarah.baatout@sckcen.be

Key words: gene expression, radiation, high-linear energy transfer, cardiovascular system, endothelial cells (cytokines) were found to be upregulated. An in silico analysis of the involved genes suggested that the transcription factors, E2F and nuclear factor (NF)- $\kappa \mathrm{B}$, may be involved in these cellular responses.

\section{Introduction}

Cardiovascular disease is considered to be one of the most important non-cancer long-term effects of ionizing radiation, as evidenced by the epidemiological data of atomic bomb survivors exposed to doses of 0.5 to $2 \mathrm{~Gy}$ (1). In the context of space exploration, high linear energy transfer (LET) radiation found in space produces high values of relative biological effectiveness (RBE), as compared to low LET radiation, such as X-rays or gamma-rays, which can increase the health risks to astronauts (2). Indeed, during long-term missions, such as a journey to Mars, astronauts are bound to be exposed to cumulative doses between 0.3 and $4 \mathrm{~Sv}$, depending on the spacecraft shielding and on the intensity of solar particle events (3).

Heavy ion irradiation is also used for terrestrial applications, such as non-conventional radiotherapy (hadron therapy), which takes advantage of the depth distribution of the dose, which is maximal at the Bragg peak, and of the increased RBE, allowing the enhanced killing effect on tumor cells while sparing the healthy tissue $(4,5)$. However, little is known of the molecular mechanisms involved in the enhanced killing properties of heavy ion irradiation. Improving our understanding of the effects of heavy ion radiation, particularly on the cardiovascular system that may be irradiated during treatment, is therefore of utmost importance for both long-term space missions and hadron therapy.

Endothelial cells are critical targets in radiation-induced cardiovascular damage $(1,6,7)$. While high doses of low LET radiation induce pro-inflammatory responses in endothelial cells, the opposite has been observed upon exposure to low doses (8-10). The mechanisms involved are not yet fully understood; however, they appear to be at least partly linked 
to the transcription factor, nuclear factor $(\mathrm{NF})-\kappa \mathrm{B}$, and the nitric oxide signaling pathway, which in turn mediates various cellular responses, including the secretion of cytokines [such as transforming growth factor (TGF)- $\beta 1$, interleukin (IL)-6, interferon (IFN)- $\gamma$, IFN- $\beta$ and tumor necrosis factor (TNF)- $\alpha$ ] and chemokines (9-11). Another possible mechanism of radiationinduced cardiovascular alteration, as shown upon low LET radiation (12-16), is the endothelial retraction and the impairment of cellular adhesion. Matrix metalloproteinases (MMPs), Rho GTPases, calcium signaling and reactive oxygen species seem to be important factors that stimulate modifications in cell junctions and the cytoskeleton through adhesion molecules and actin (12-16). Although high LET radiation has been shown to reduce the length of a 3D human endothelial vessel model, both developing and mature (17), only a few studies have been conducted to identify the mechanisms involved in the endothelial response to high LET radiation $(18,19)$.

Thus, the aim of this study was to investigate the effects of moderate and high doses of high LET nickel ion (Ni) irradiation on gene expression in endothelial cells in order to elucidate the molecular mechanisms responsible for radiation-induced cardiovascular damage. For this purpose, the EA.hy926 cell line, which originates from human umbilical vein endothelial cells, was irradiated with nickel ions (LET, $183 \mathrm{keV} / \mu \mathrm{m}$ ) at moderate (0.5 Gy) and high (2 and 5 Gy) doses after which gene expression was determined by whole-genome microarray analysis.

\section{Materials and methods}

Cell culture. The human EA.hy926 endothelial cells were obtained from the American Type Culture Collection (ATCC; Manassas, VA, USA). They were cultured $\left(37^{\circ} \mathrm{C}-5 \% \mathrm{CO}_{2}\right)$ in Dulbecco's modified Eagle's medium supplemented with $10 \%$ fetal bovine serum and $1 \%$ penicillin/streptomycin (all from N.V. Invitrogen S.A., Merelbeke, Belgium). The cells were regularly examined for the absence of mycoplasma using the LookOut ${ }^{\circledR}$ Mycoplasma PCR Detection kit (Sigma-Aldrich, St. Louis, MO, USA).

Nickel irradiation. The cells were seeded at a density of $10^{5}$ cells in $12.5 \mathrm{~cm}^{2}$ flasks. Twenty-four hours after plating, the flasks were placed in a transportable incubator $\left(37^{\circ} \mathrm{C}\right)$ and moved from the resident laboratory (Mol, Belgium) to the GSI Helmholtzzentrum für Schwerionenforschung $\mathrm{GmbH}$ (Darmstadt, Germany). Forty-eight hours after plating, the subconfluent cells were irradiated in flasks completely filled with culture medium with a $1 \mathrm{GeV} / \mathrm{u}$ Ni beam at the SIS facility at GSI with the intensity controlled raster scanning technique as described by Haberer et al (20). The ion energy at the sample position was approximately $930 \mathrm{MeV} / \mathrm{u}$ with a LET of $183 \mathrm{keV} / \mu \mathrm{m}$ (calculated with the program code ATIMA). The culture flasks were placed vertically and exposed perpendicularly to the nickel ion beam at the following doses: 0.5, 2 and 5 Gy. Non-irradiated control samples were treated similarly to the irradiated samples, but placed out of the beam. Following irradiation, the cells were incubated $\left(37^{\circ} \mathrm{C}, 5 \% \mathrm{CO}_{2}\right)$ in $2 \mathrm{ml}$ of conditioned medium until fixation time points $(2,8$ and $24 \mathrm{~h})$.

DNA double-strand break detection (detection of $\gamma$-H2AX foci). The cells were fixed in $4 \%$ paraformaldehyde (Merck KGaA,
Darmstadt, Germany) 2 and $24 \mathrm{~h}$ after irradiation. They were then treated with $0.25 \%$ Triton $\mathrm{X}$ for 5 min, blocked with $3 \%$ bovine serum albumin (both from Sigma-Aldrich) for $30 \mathrm{~min}$ and incubated overnight with mouse anti- $\gamma-\mathrm{H} 2 \mathrm{AX}$ antibody (Abcam, Cambridge, MA, USA) at $4^{\circ} \mathrm{C}$. After a second blocking of $10 \mathrm{~min}$, the cells were incubated for $1 \mathrm{~h}$ with anti-mouse secondary antibody coupled to FITC (SigmaAldrich) at $37^{\circ} \mathrm{C}$ and then mounted in Vectashield mounting medium (Vector Laboratories, Burlingame, CA, USA) with DAPI. Between each of the previous steps, the slides were washed with phosphate-buffered saline (PBS).

An automated inverted fluorescence microscope (TE2000-E; Nikon, Tokyo, Japan), equipped with a motorized XYZ stage, emission and excitation filter wheels, shutters and a triple dichroic mirror (436/514/604) was used for the image acquisition of the immunostained slides. Images were acquired with a 40X Plan Fluor oil objective (NA 1.3) and an Andor iXon EMCCD camera (Andor Technology, South Windsor, CT, USA). For each sample, at least 12 fields were acquired on $5 \mathrm{z}$-stack focusses $(1 \mu \mathrm{m})$. The $\gamma$-H2AX spot number and spot occupancy were analyzed with the INSCYDE plugin for ImageJ as previously described (21). Spot occupancy was defined for each nucleus as the sum of the spot areas divided by the nucleus area (spot_occupancy = sum (spot_area)/ nuclear_area). A minimum number of 100 cells was analyzed in 2 biological replicates per condition. For statistical analyses, the data were analyzed using the Mann-Whitney U test with SPSS version 17.0 software (IBM Corp., Chicago, IL, USA) and box plots were generated using the same software. P-values $<0.05$ were considered to indicate statistically significant differences.

RNA extraction. At 2 time points after irradiation (8 and $24 \mathrm{~h}$ ), the adherent cells were washed in PBS, lysed in $350 \mathrm{ml}$ of AllPrep DNA/RNA/Protein Mini kit lysis buffer (Qiagen, Hilden, Germany) and frozen at $-80^{\circ} \mathrm{C}$. RNA was extracted using the same kit and its concentration was measured using a NanoDrop spectrophotometer (Thermo Fisher Scientific, Waltham, MA, USA), while its quality (RNA integrity number, RIN) was determined using Agilent's lab-on-chip Bioanalyzer 2100 (Agilent Technologies, Santa Clara, CA, USA). All RNA samples had a RIN value $>9.0$.

Affymetrix microarrays and data analysis. RNA was processed using the GeneChip WT cDNA Synthesis and Amplification kit (Affymetrix, Santa Clara, CA, USA) according to the manufacturer's instructions. The resulting RNA was hybridized to Affymetrix Human Gene 1.0 ST arrays which contain an estimated number of 28,869 genes based on the March 2006 [UCSC $\mathrm{Hg}$ 18; National Center for Biotechnology Information (NCBI) build 36] human genome assembly. Biological triplicates were collected for each condition.

Raw data (.cel-files) were imported at exon level in Partek Genomics Suite version 6.5 (Partek, Inc., St. Louis, MO, USA). Briefly, robust multi-array average (RMA) background correction was applied, data were normalized by quantile normalization and probeset summarization was performed by the median polish method. Gene summarization was performed using one-step Tukey's biweight method. The obtained data were analyzed with Partek Genomics Suite for single gene analysis. One- or two-way ANOVA, taking into consideration 

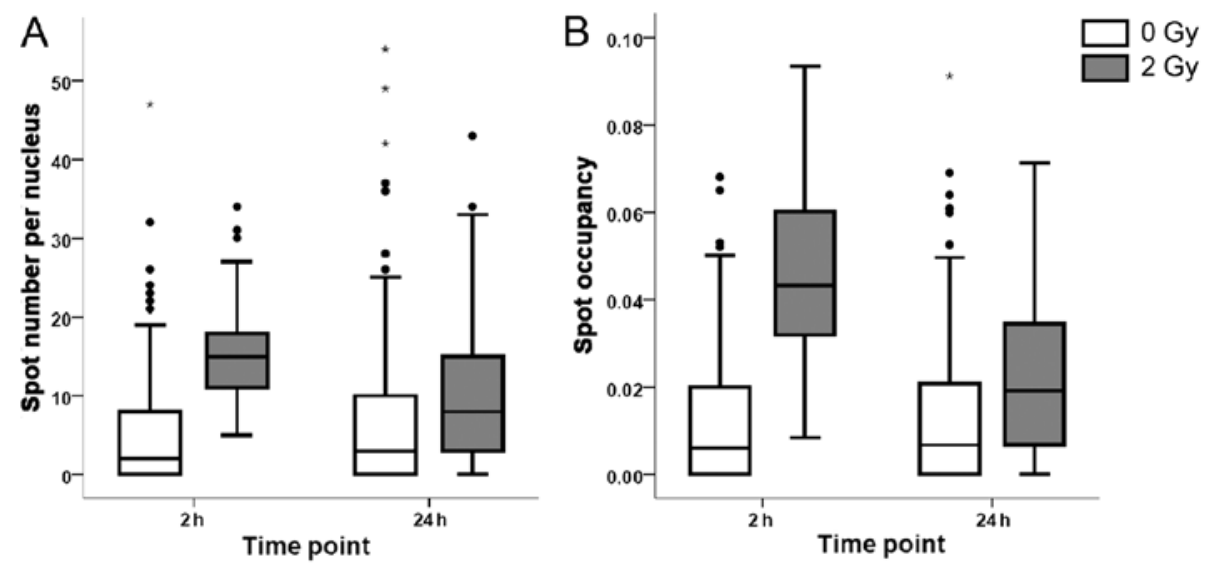

Figure 1. Box plots of the $\gamma$-H2AX (A) spot number per nucleus and (B) spot occupancy in human endothelial cells 2 and $24 \mathrm{~h}$ after irradiation with nickel ions (Ni). Dots represent outliers and stars represent extreme values. In both graphs, the spot number in the samples subjected to $2 \mathrm{~Gy}$ irradation (gray boxes) was significantly higher than the controls ( $0 \mathrm{~Gy}$, white boxes), as shown by the Mann-Whitney U test.

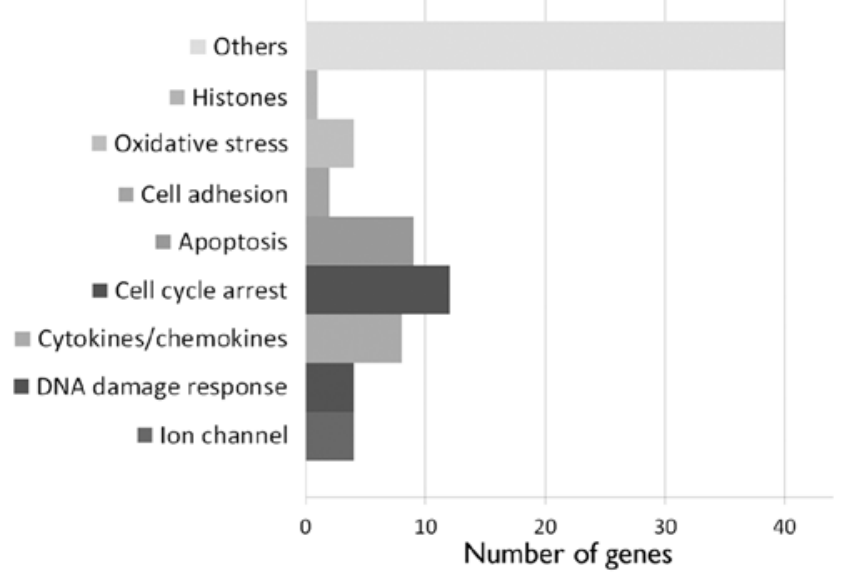

Figure 2. Number of upregulated genes in specific cellular processes $24 \mathrm{~h}$ after nickel ion irradiation ( $5 \mathrm{~Gy}$ ). The referred processes were determined by search on the National Center for Biotechnology Information (NCBI) database.

the scan date (where applicable) and the dose as factors, were performed for each time point. In order to determine statistical significance, thresholds were set on the p-value $<0.001$ and on the fold-change $>1.5$.

The enrichment of the transcription factor binding motifs was analyzed using Pscan Ver. 1.2 (22) and the JASPAR database, scanning in a region from -950 to +50 base pairs from the transcription start site.

\section{Results}

DNA damage. To assess DNA damage induction by nickel ion irradiation and evaluate the cell capacity required to repair this damage, we performed a high content cytometric assay of $\gamma-\mathrm{H} 2 \mathrm{AX}, 2$ and $24 \mathrm{~h}$ after exposure. As measured by the number of $\gamma$-H2AX foci, DNA damage was significantly increased $2 \mathrm{~h}$ after nickel ion irradiation ( $2 \mathrm{~Gy}$ ), with an average number of 15 foci per nucleus vs. 2 foci per nucleus in the control samples (Fig. 1A). Twenty-four hours after irradiation, the number of foci decreased to 9 per nucleus, which was significantly higher than the values of controls, indicating that part of the DNA damage persisted for at least $24 \mathrm{~h}$. Similar trends were observed for the spot occupancy, which is the fraction of the projected area of the nucleus occupied by the signal from the $\gamma$-H2AX foci (Fig. 1B).

Effects of nickel irradiation on gene expression. In order to evaluate gene expression, we performed microarrays 8 and $24 \mathrm{~h}$ after irradiation. A 0.5 Gy irradiation, both after 8 and $24 \mathrm{~h}$, elicited a subtle effect on gene expression in the EA.hy926 cells. Six annotated genes were differentially regulated with fold changes (FC) between 1.5 and 1.8 after $8 \mathrm{~h}$, and 18 genes were differentially regulated with an FC between 1.5 and 2.3 after $24 \mathrm{~h}$. A more drastic effect was observed at $5 \mathrm{~Gy}, 24 \mathrm{~h}$ after irradiation. At this time point, we detected the upregulation of 77 annotated genes (Fig. 2 and Table I; maximum FC, 3.4). Among these genes, cytokines and chemokines (CXCL5, TGFA, TRIM22, TNFSF9, EBI3, IL-6, IL-11 and CD70) were identified, as well as genes involved in DNA damage response (SPATA18, POLL, APOBEC3H and SESN1), cell cycle arrest (ZMAT3, MXD4, TP53INP1, HSPB8, TGFA, SESN2, BTG2, DTX3, TOB1, HBP1, CDKN1A and PLK3) and apoptosis (TP53INP1, HSPB8, TGFA, TP53I3, MOAP1, CYFIP2, TRADD, DTX3 and FAS). In addition, we observed the upregulation of genes coding for ion channels (SLC22A4, KCNJ2, ORAI3 and CLIC3), cell adhesion (CEACAM1 and NEU1) and oxidative stress response proteins (FMO4, FDXR, SIRT2 and SESN1).

A total of 145 annotated genes was downregulated $24 \mathrm{~h}$ after nickel ion irradiation (5 Gy) (Fig. 3 and Table I). The majority (62 genes) is known to be involved in various aspects of cell division, such as DNA replication, replication forks and chromosome assembly and segregation (Table II and Fig. 4). Other downregulated genes found have been implicated in post-replicative DNA repair (UNG, UPF3A, MSH2 and MSH6), nucleotide biosynthesis (DHFR and RRM2), DNA repair (FANCA, MMS22L, NFKBIL2, RAD51, EXO1 and HMGB2), positive (YAP1) and negative regulation of apoptosis (DHRS2, DHCR24 and MTBP), Rho signaling (ARHGAP19, ARHGAP11B and RACGAP1) and cell adhesion (PVRL1 and DLGAP5). 
Table I. List of the differentially expressed genes at 8 and $24 \mathrm{~h}$ after 0.5 and $5 \mathrm{~Gy}$ of nickel ion irradiation.

\begin{tabular}{|c|c|c|c|c|c|c|c|}
\hline \multicolumn{4}{|c|}{ Downregulated genes } & \multicolumn{4}{|c|}{ Upregulated genes } \\
\hline Gene symbol & GenBank & p-value & $\mathrm{FC}$ & Gene symbol & GenBank & p-value & $\mathrm{FC}$ \\
\hline \multicolumn{8}{|c|}{ List of differentially expressed genes $8 \mathrm{~h}$ after $0.5 \mathrm{~Gy}$ nickel ion irradiation } \\
\hline CRYBB2 & NM_000496 & $1,02 \mathrm{E}-03$ & $-1,800$ & HSP90AA6P & NR_036751 & $6,55 \mathrm{E}-03$ & 1,630 \\
\hline GNAT1 & NM_144499 & $5,54 \mathrm{E}-03$ & $-1,601$ & RFT1 & NM_052859 & $6,83 \mathrm{E}-03$ & 1,572 \\
\hline DNAJB13 & NM_153614 & $3,79 \mathrm{E}-03$ & $-1,521$ & DEFB123 & NM_153324 & $8,50 \mathrm{E}-03$ & 1,518 \\
\hline \multicolumn{8}{|c|}{ List of differentially expressed genes $24 \mathrm{~h}$ after $0.5 \mathrm{~Gy}$ nickel-ion irradiation } \\
\hline UPF3A & NM_023011 & $1,53 \mathrm{E}-03$ & $-1,932$ & C1orf113 & ENST00000312808 & $8,96 \mathrm{E}-03$ & 2,224 \\
\hline $\mathrm{E} 2 \mathrm{~F}^{\mathrm{a}}$ & NM_024680 & $5,38 \mathrm{E}-03$ & $-1,716$ & SNORD53 & NR_002741 & $6,78 \mathrm{E}-03$ & 2,182 \\
\hline LIMA1 & NM_001113546 & $9,63 \mathrm{E}-03$ & $-1,670$ & SLC45A4 & ВC033223 & $8,10 \mathrm{E}-03$ & 2,049 \\
\hline C16orf55 & AK303024 & $7,00 \mathrm{E}-04$ & $-1,600$ & HIST1H2BD & NM_021063 & $9,01 \mathrm{E}-03$ & 2,028 \\
\hline HLA-DRB4 & AK293020 & $3,18 \mathrm{E}-03$ & $-1,583$ & ZNF16 & NM_001029976 & $3,65 \mathrm{E}-03$ & 1,952 \\
\hline $\mathrm{MCM}^{\mathrm{a}}$ & NM_182751 & $9,77 \mathrm{E}-03$ & $-1,530$ & RNF207 & NM_207396 & $7,07 \mathrm{E}-03$ & 1,677 \\
\hline \multirow[t]{5}{*}{ PROK2 } & NM_001126128 & $8,29 \mathrm{E}-03$ & $-1,518$ & $\mathrm{LCE}_{1 \mathrm{E}}^{\mathrm{b}}$ & NM_178353 & 4,89E-03 & 1,619 \\
\hline & & & & C10orf72 & NM_001031746 & $6,04 \mathrm{E}-04$ & 1,592 \\
\hline & & & & $\mathrm{PMCH}$ & NM_002674 & 7,49E-03 & 1,591 \\
\hline & & & & RUNDC3B & NM_138290 & $1,98 \mathrm{E}-03$ & 1,571 \\
\hline & & & & $\mathrm{ORAI}^{\mathrm{b}}$ & NM_152288 & $7,01 \mathrm{E}-03$ & 1,515 \\
\hline \multicolumn{8}{|c|}{ List of differentially expressed genes $24 \mathrm{~h}$ after 5 Gy nickel-ion irradiation } \\
\hline 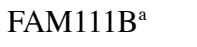 & NM_198947 & $5,65 \mathrm{E}-03$ & $-5,894$ & $\mathrm{ACTA} 2^{\mathrm{b}}$ & NM_001141945 & $2,01 \mathrm{E}-05$ & 3,424 \\
\hline $\mathrm{PCBP}^{\mathrm{a}}$ & NM_006196 & $6,05 \mathrm{E}-04$ & $-3,611$ & TP53INP1 & NM_033285 & $1,02 \mathrm{E}-04$ & 2,976 \\
\hline DHRS2 & NM_182908 & $6,48 \mathrm{E}-05$ & $-3,090$ & $\mathrm{CD} 70^{\mathrm{b}}$ & NM_001252 & $9,56 \mathrm{E}-03$ & 2,702 \\
\hline MCM6 $^{\mathrm{a}}$ & NM_005915 & $1,81 \mathrm{E}-05$ & $-3,069$ & PHOSPHO1 & NM_001143804 & $9,47 \mathrm{E}-04$ & 2,629 \\
\hline HIST1H1T & NM_005323 & $6,99 \mathrm{E}-03$ & $-2,762$ & CDKN1A & NR_037151 & $8,27 \mathrm{E}-03$ & 2,221 \\
\hline ZNF367 & NM_153695 & $1,61 \mathrm{E}-04$ & $-2,669$ & CEACAM1 & NM_001712 & $5,56 \mathrm{E}-04$ & 2,184 \\
\hline KIF20A & NM_005733 & 4,17E-04 & $-2,595$ & $\mathrm{BTG} 2^{\mathrm{b}}$ & NM_006763 & $1,08 \mathrm{E}-03$ & 2,170 \\
\hline LMNB1 & NM_005573 & $9,94 \mathrm{E}-04$ & $-2,561$ & APOBEC $3 \mathrm{H}^{\mathrm{b}}$ & NM_001166003 & 4,32E-03 & 2,070 \\
\hline HIST1H1D & NM_005320 & $4,45 \mathrm{E}-03$ & $-2,478$ & TRIM22 ${ }^{\mathrm{b}}$ & NM_006074 & $4,27 \mathrm{E}-04$ & 2,063 \\
\hline $\mathrm{E} 2 \mathrm{~F} 8^{\mathrm{a}}$ & NM_024680 & $3,88 \mathrm{E}-04$ & $-2,469$ & $\mathrm{KCNJ} 2^{\mathrm{b}}$ & NM_000891 & $1,88 \mathrm{E}-03$ & 2,055 \\
\hline HAUS8 & NM_033417 & $1,71 \mathrm{E}-05$ & $-2,444$ & SPATA $18^{\mathrm{b}}$ & NM_145263 & $1,16 \mathrm{E}-06$ & 2,026 \\
\hline MYBL2 & NM_002466 & $7,56 \mathrm{E}-04$ & $-2,369$ & ZNF223 ${ }^{b}$ & NM_013361 & $4,56 \mathrm{E}-03$ & 1,997 \\
\hline $\mathrm{UHRF}^{\mathrm{a}}$ & NM_001048201 & $5,93 \mathrm{E}-03$ & $-2,363$ & $\mathrm{LCE} 1 \mathrm{E}^{\mathrm{b}}$ & NM_178353 & $8,26 \mathrm{E}-04$ & 1,986 \\
\hline SRP19 & ENST00000512790 & $6,66 \mathrm{E}-03$ & $-2,337$ & FDXR & NM_024417 & $1,72 \mathrm{E}-03$ & 1,972 \\
\hline UPF3A & NM_023011 & $4,54 \mathrm{E}-04$ & $-2,292$ & TUBA4A & NM_006000 & $1,36 \mathrm{E}-03$ & 1,932 \\
\hline DLGAP5 $^{a}$ & NM_014750 & $9,85 \mathrm{E}-03$ & $-2,264$ & PSTPIP2 & NM_024430 & $5,51 \mathrm{E}-04$ & 1,924 \\
\hline $\mathrm{ATAD}^{\mathrm{a}}$ & NM_014109 & 7,92E-03 & $-2,239$ & $\mathrm{ACY}^{\mathrm{b}}$ & NM_080658 & $8,81 \mathrm{E}-03$ & 1,922 \\
\hline $\mathrm{UNG}^{\mathrm{a}}$ & NM_003362 & $2,21 \mathrm{E}-05$ & $-2,220$ & SLC40A1 ${ }^{\mathrm{b}}$ & NM_014585 & $2,55 \mathrm{E}-03$ & 1,921 \\
\hline HELLS $^{a}$ & NM_018063 & $8,16 \mathrm{E}-03$ & $-2,194$ & TMEM150A & NM_001031738 & $5,23 \mathrm{E}-04$ & 1,904 \\
\hline $\mathrm{MCM}^{\mathrm{a}}$ & NM_002388 & $1,81 \mathrm{E}-03$ & $-2,189$ & MXD4 & NM_006454 & $1,09 \mathrm{E}-04$ & 1,903 \\
\hline FIGNL1 & NM_001042762 & $6,39 \mathrm{E}-03$ & $-2,184$ & IL-6 ${ }^{\mathrm{b}}$ & NM_000600 & 2,89E-03 & 1,883 \\
\hline KIF11 & NM_004523 & $9,41 \mathrm{E}-03$ & $-2,164$ & NCRNA00219 & NR_015370 & $8,85 \mathrm{E}-03$ & 1,864 \\
\hline FBXO5 & NM_012177 & $1,38 \mathrm{E}-05$ & $-2,150$ & $\mathrm{KBTBD}^{\mathrm{b}}$ & NM_032505 & $8,57 \mathrm{E}-03$ & 1,833 \\
\hline $\mathrm{E} 2 \mathrm{~F} 2^{\mathrm{a}}$ & NM_004091 & $3,11 \mathrm{E}-04$ & $-2,126$ & NIPAL $^{b}$ & NM_020448 & $1,16 \mathrm{E}-06$ & 1,819 \\
\hline WDR76 & NM_024908 & $4,11 \mathrm{E}-04$ & $-2,108$ & RAB4B & NM_016154 & $3,30 \mathrm{E}-03$ & 1,811 \\
\hline 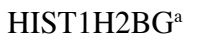 & NM_003518 & $1,90 \mathrm{E}-03$ & $-2,106$ & SAT $1^{\text {b }}$ & NR_027783 & $7,52 \mathrm{E}-03$ & 1,810 \\
\hline LOC 1720 & NR_033423 & $1,54 \mathrm{E}-03$ & $-2,101$ & SLC22A4 & NM_003059 & 7,67E-04 & 1,796 \\
\hline CAMK2N1 & NM_018584 & $1,24 \mathrm{E}-03$ & $-2,091$ & NEU1 & NM_000434 & $2,68 \mathrm{E}-03$ & 1,778 \\
\hline DLEU2 $^{\mathrm{a}}$ & NR_002612 & $6,87 \mathrm{E}-03$ & $-2,088$ & $\mathrm{CYFIP}^{\mathrm{b}}$ & NM_001037332 & $5,48 \mathrm{E}-03$ & 1,770 \\
\hline $\mathrm{MCM}^{\mathrm{a}}$ & NM_006739 & $2,95 \mathrm{E}-04$ & $-2,084$ & TNFSF9 & NM_003811 & 7,09E-04 & 1,736 \\
\hline ANKRD36B & NM_025190 & $3,63 \mathrm{E}-03$ & $-2,082$ & TMEM $217^{\mathrm{b}}$ & NM_145316 & $7,60 \mathrm{E}-03$ & 1,730 \\
\hline POLA $^{\mathrm{a}}$ & NM_016937 & $2,54 \mathrm{E}-03$ & $-2,073$ & IL-11 & NM_000641 & $3,38 \mathrm{E}-03$ & 1,721 \\
\hline BUB1B & NM_001211 & $1,55 \mathrm{E}-03$ & $-1,995$ & ATP6V0A4 & NM_020632 & 7,57E-03 & 1,712 \\
\hline GPSM2 & NM_013296 & $2,43 \mathrm{E}-04$ & $-1,988$ & FMO4 & NM_002022 & $1,05 \mathrm{E}-03$ & 1,707 \\
\hline
\end{tabular}


Table I. Continued.

\begin{tabular}{|c|c|c|c|c|c|c|c|}
\hline \multicolumn{4}{|c|}{ Downregulated genes } & \multicolumn{4}{|c|}{ Upregulated genes } \\
\hline Gene symbol & GenBank & p-value & FC & Gene symbol & GenBank & p-value & $\mathrm{FC}$ \\
\hline HMGB2 & NM_001130688 & $3,20 \mathrm{E}-03$ & $-1,979$ & WIPI1 & NM_017983 & 7,41E-04 & 1,705 \\
\hline DHCR24 & NM_014762 & $3,24 \mathrm{E}-04$ & $-1,970$ & HBP1 & NM_012257 & $7,81 \mathrm{E}-03$ & 1,684 \\
\hline $\mathrm{MCM}^{\mathrm{a}}$ & NM_005916 & $2,60 \mathrm{E}-05$ & $-1,956$ & $\mathrm{UCN} 2$ & NM_033199 & $4,81 \mathrm{E}-03$ & 1,683 \\
\hline ANLN & NM_018685 & $7,33 \mathrm{E}-03$ & $-1,953$ & bEBI3 & NM_005755 & $1,99 \mathrm{E}-03$ & 1,676 \\
\hline NDC80 & NM_006101 & $2,04 \mathrm{E}-04$ & $-1,953$ & bFAS & NM_000043 & $9,18 \mathrm{E}-03$ & 1,673 \\
\hline $\mathrm{MCM}^{\mathrm{a}}$ & NM_004526 & $2,05 \mathrm{E}-04$ & $-1,927$ & CLIC $^{\mathrm{b}}$ & NM_004669 & $8,25 \mathrm{E}-03$ & 1,666 \\
\hline CDKN3 & NM_005192 & $9,25 \mathrm{E}-03$ & $-1,917$ & TGFA & NM_003236 & $1,70 \mathrm{E}-04$ & 1,662 \\
\hline EMP2 & NM_001424 & $1,87 \mathrm{E}-03$ & $-1,915$ & $\mathrm{NCF} 2^{\mathrm{b}}$ & NM_000433 & $6,46 \mathrm{E}-03$ & 1,656 \\
\hline TACC3 & NM_006342 & $2,72 \mathrm{E}-03$ & $-1,906$ & NADSYN1 & NM_018161 & 4,99E-04 & 1,651 \\
\hline LHX2 & NM_004789 & $8,33 \mathrm{E}-03$ & $-1,883$ & $\mathrm{CXCL5}^{\mathrm{b}}$ & NM_002994 & $2,65 \mathrm{E}-05$ & 1,643 \\
\hline NCAPG2 & NM_017760 & 1,59E-03 & $-1,881$ & SESN2 ${ }^{\mathrm{b}}$ & NM_031459 & $8,22 \mathrm{E}-04$ & 1,642 \\
\hline DHFR & NM_000791 & $1,91 \mathrm{E}-05$ & $-1,878$ & HSPB8 & NM_014365 & $1,53 \mathrm{E}-04$ & 1,634 \\
\hline PER3 $^{a}$ & NM_016831 & $8,02 \mathrm{E}-03$ & $-1,867$ & 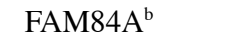 & NM_145175 & 3,91E-04 & 1,623 \\
\hline SEMA3D & NM_152754 & $7,14 \mathrm{E}-03$ & $-1,867$ & $\mathrm{ORAI}^{\mathrm{b}}$ & NM_152288 & $3,54 \mathrm{E}-03$ & 1,616 \\
\hline KIFC1 & NM_002263 & $1,96 \mathrm{E}-04$ & $-1,860$ & C9orf150 & NM_203403 & $2,10 \mathrm{E}-03$ & 1,614 \\
\hline DEPDC1B & NM_018369 & $5,71 \mathrm{E}-03$ & $-1,853$ & C2orf80 & NM_001099334 & $5,67 \mathrm{E}-04$ & 1,613 \\
\hline USP1 & NM_003368 & $1,74 \mathrm{E}-03$ & $-1,848$ & PLK3 & NM_004073 & $8,79 \mathrm{E}-03$ & 1,611 \\
\hline CCNE2 & NM_057749 & $1,34 \mathrm{E}-03$ & $-1,841$ & ${\text { MAGED } 4^{\mathrm{b}}}$ & NM_001098800 & $1,62 \mathrm{E}-03$ & 1,611 \\
\hline PRC1 & NM_003981 & $1,06 \mathrm{E}-03$ & $-1,838$ & LRRC29 & NM_012163 & $2,25 \mathrm{E}-06$ & 1,589 \\
\hline DEPDC1 & NM_001114120 & $1,89 \mathrm{E}-04$ & $-1,819$ & POLL & NM_001174084 & $2,96 \mathrm{E}-03$ & 1,583 \\
\hline ORC1 & NM_004153 & $2,50 \mathrm{E}-03$ & $-1,811$ & DFNA5 & NM_004403 & $6,35 \mathrm{E}-04$ & 1,578 \\
\hline $\mathrm{CDCA}^{\mathrm{a}}$ & NM_031942 & $1,42 \mathrm{E}-04$ & $-1,807$ & CRYAB & NM_001885 & $3,49 \mathrm{E}-04$ & 1,577 \\
\hline MCM10a & NM_182751 & $2,11 \mathrm{E}-03$ & $-1,801$ & WBP5 & NM_016303 & $2,50 \mathrm{E}-04$ & 1,569 \\
\hline $\mathrm{CDT}^{\mathrm{a}}$ & NM_030928 & $5,55 \mathrm{E}-03$ & $-1,800$ & SESN1 & NM_014454 & $6,34 \mathrm{E}-03$ & 1,565 \\
\hline 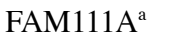 & NM_022074 & $5,61 \mathrm{E}-04$ & $-1,798$ & $\mathrm{RDH}_{10} 0^{\mathrm{b}}$ & NM_172037 & $8,25 \mathrm{E}-03$ & 1,556 \\
\hline STX11 ${ }^{\mathrm{a}}$ & NM_003764 & $1,61 \mathrm{E}-03$ & $-1,795$ & BHLHE40 ${ }^{\mathrm{b}}$ & NM_003670 & $9,87 \mathrm{E}-03$ & 1,553 \\
\hline $\mathrm{MSH}^{\mathrm{a}}$ & NM_000251 & $7,76 \mathrm{E}-04$ & $-1,794$ & FAM113A & AK293638 & 7,81E-04 & 1,550 \\
\hline MKKS & NM_018848 & $6,55 \mathrm{E}-04$ & $-1,794$ & LOC100130581 & NR_027413 & $6,57 \mathrm{E}-03$ & 1,546 \\
\hline CEP78 & NM_001098802 & $9,07 \mathrm{E}-03$ & $-1,790$ & MOAP1 & NM_022151 & 2,89E-04 & 1,543 \\
\hline RFC4 & NM_002916 & $3,92 \mathrm{E}-03$ & $-1,789$ & TP53I $^{\mathrm{b}}$ & NM_004881 & $1,71 \mathrm{E}-04$ & 1,543 \\
\hline KIF23 & NM_138555 & $3,79 \mathrm{E}-03$ & $-1,789$ & OR51B6 & NM_001004750 & $5,49 \mathrm{E}-03$ & 1,542 \\
\hline MLF1IP ${ }^{a}$ & NM_024629 & $2,81 \mathrm{E}-04$ & $-1,785$ & NIPSNAP $1^{\mathrm{b}}$ & NM_003634 & $3,88 \mathrm{E}-04$ & 1,541 \\
\hline CEP55 & NM_018131 & 4,87E-04 & $-1,782$ & HHAT $^{\mathrm{b}}$ & NM_001170580 & $2,41 \mathrm{E}-03$ & 1,536 \\
\hline TCF19a & NM_007109 & $6,78 \mathrm{E}-04$ & $-1,781$ & ARR3 & NM_004312 & 7,01E-04 & 1,535 \\
\hline BUB1 & NM_004336 & $6,08 \mathrm{E}-04$ & $-1,780$ & $\mathrm{SIRT}^{\mathrm{b}}$ & NM_012237 & $4,30 \mathrm{E}-03$ & 1,533 \\
\hline CHAF1B & NM_005441 & $1,22 \mathrm{E}-03$ & $-1,773$ & C15orf $33^{b}$ & NM_152647 & $1,43 \mathrm{E}-03$ & 1,526 \\
\hline $\mathrm{EZH} 2^{\mathrm{a}}$ & NM_004456 & $5,38 \mathrm{E}-04$ & $-1,772$ & $\mathrm{RBKS}^{\mathrm{b}}$ & NM_022128 & $5,82 \mathrm{E}-03$ & 1,523 \\
\hline PLK4 & NM_014264 & $2,16 \mathrm{E}-04$ & $-1,757$ & DTX $^{b}$ & NM_178502 & $4,95 \mathrm{E}-03$ & 1,519 \\
\hline $\mathrm{E} 2 \mathrm{~F} 1^{\mathrm{a}}$ & NM_005225 & $1,61 \mathrm{E}-03$ & $-1,755$ & TOB1 & NM_005749 & $6,18 \mathrm{E}-03$ & 1,517 \\
\hline $\mathrm{H}_{1 \mathrm{~F} 0}{ }^{\mathrm{a}}$ & NM_005318 & $1,51 \mathrm{E}-04$ & $-1,740$ & $\mathrm{ADCY} 4^{\mathrm{b}}$ & NM_001198592 & $5,24 \mathrm{E}-03$ & 1,514 \\
\hline CEP57L1 & NM_001083535 & $5,12 \mathrm{E}-03$ & $-1,739$ & ARL15 & NM_019087 & $1,98 \mathrm{E}-03$ & 1,512 \\
\hline NUSAP1 $^{\mathrm{a}}$ & NM_016359 & $8,15 \mathrm{E}-05$ & $-1,730$ & TRADD $^{\mathrm{b}}$ & NM_003789 & 2,41E-03 & 1,509 \\
\hline ESPL1 & NM_012291 & $9,12 \mathrm{E}-03$ & $-1,724$ & ZMAT3b $^{\mathrm{b}}$ & NM_022470 & $5,71 \mathrm{E}-05$ & 1,502 \\
\hline KIF2C & NM_006845 & $7,54 \mathrm{E}-04$ & $-1,718$ & & & & \\
\hline DEPDC4 & NM_152317 & $3,25 \mathrm{E}-03$ & $-1,714$ & & & & \\
\hline MSH6 & NM_000179 & $6,14 \mathrm{E}-03$ & $-1,712$ & & & & \\
\hline $\mathrm{CDC}^{\mathrm{a}}$ & NM_001254 & $2,07 \mathrm{E}-03$ & $-1,710$ & & & & \\
\hline PM20D2 $^{\mathrm{a}}$ & NM_001010853 & $7,65 \mathrm{E}-04$ & $-1,706$ & & & & \\
\hline PVRL1 & NM_002855 & $9,24 \mathrm{E}-03$ & $-1,693$ & & & & \\
\hline C16orf55 & AK303024 & $3,86 \mathrm{E}-04$ & $-1,691$ & & & & \\
\hline RRM2a & NM_001165931 & 7,98E-03 & $-1,687$ & & & & \\
\hline
\end{tabular}


Table I. Continued.

\begin{tabular}{|c|c|c|c|c|c|c|c|}
\hline \multicolumn{4}{|c|}{ Downregulated genes } & \multicolumn{4}{|c|}{ Downregulated genes } \\
\hline Gene Symbol & GenBank & p-value & $\mathrm{FC}$ & Gene Symbol & GenBank & p-value & FC \\
\hline HIST1H3F ${ }^{a}$ & NM_021018 & $8,20 \mathrm{E}-03$ & $-1,682$ & DDX12 & NR_033399 & $3,55 \mathrm{E}-03$ & $-1,570$ \\
\hline ZNF716 & NM_001159279 & 2,70E-04 & $-1,682$ & FLJ30064 & AK054626 & $2,40 \mathrm{E}-03$ & $-1,562$ \\
\hline KIAA1524 & NM_020890 & 1,99E-04 & $-1,680$ & $\mathrm{USP} 7^{\mathrm{a}}$ & NM_020935 & $3,15 \mathrm{E}-04$ & $-1,566$ \\
\hline FANCA $^{a}$ & NM_000135 & $6,54 \mathrm{E}-06$ & $-1,680$ & PLS1 & NM_001172312 & $9,23 \mathrm{E}-04$ & $-1,559$ \\
\hline KLHL23 & NM_144711 & $8,07 \mathrm{E}-03$ & $-1,679$ & MT4 & NM_032935 & $7,81 \mathrm{E}-03$ & $-1,558$ \\
\hline CDCA2 & NM_152562 & $3,22 \mathrm{E}-03$ & $-1,677$ & GTSE1 & NM_016426 & $6,65 \mathrm{E}-03$ & $-1,556$ \\
\hline WHSC1 & NM_133330 & $7,07 \mathrm{E}-04$ & $-1,677$ & $\mathrm{KCTD} 12^{\mathrm{a}}$ & NM_138444 & $7,18 \mathrm{E}-03$ & $-1,555$ \\
\hline MMS22L & NM_198468 & $2,44 \mathrm{E}-04$ & $-1,675$ & ZNF749a & NM_001023561 & $3,89 \mathrm{E}-03$ & $-1,553$ \\
\hline FAM72D & AB096683 & $2,28 \mathrm{E}-03$ & $-1,674$ & $\mathrm{CENPH}^{\mathrm{a}}$ & NM_022909 & $1,88 \mathrm{E}-03$ & $-1,547$ \\
\hline KIAA0101 ${ }^{\mathrm{a}}$ & NM_014736 & 2,64E-03 & $-1,663$ & DDX11 & NM_030653 & $7,35 \mathrm{E}-04$ & $-1,545$ \\
\hline AREG & NM_001657 & $8,49 \mathrm{E}-03$ & $-1,658$ & SNX5 & NM_152227 & $4,96 \mathrm{E}-03$ & $-1,543$ \\
\hline $\mathrm{GINS}^{\mathrm{a}}$ & NM_016095 & $7,86 \mathrm{E}-03$ & $-1,657$ & $\mathrm{MTBP}^{\mathrm{a}}$ & NM_022045 & $4,20 \mathrm{E}-03$ & $-1,539$ \\
\hline ARHGAP11B ${ }^{\mathrm{a}}$ & NM_001039841 & $1,80 \mathrm{E}-03$ & $-1,657$ & GAR1 & NM_018983 & $8,34 \mathrm{E}-03$ & $-1,539$ \\
\hline LYAR & NM_017816 & $9,45 \mathrm{E}-03$ & $-1,656$ & NUF2 & NM_145697 & $2,74 \mathrm{E}-04$ & $-1,531$ \\
\hline YAP1 & NM_001130145 & $1,60 \mathrm{E}-03$ & $-1,655$ & $\mathrm{CCNF}^{\mathrm{a}}$ & NM_001761 & $8,64 \mathrm{E}-04$ & $-1,529$ \\
\hline PKP4 & NM_003628 & 4,89E-03 & $-1,653$ & $\mathrm{PBK}^{\mathrm{a}}$ & NM_018492 & $3,92 \mathrm{E}-03$ & $-1,528$ \\
\hline FGF12 & NM_021032 & $2,85 \mathrm{E}-03$ & $-1,649$ & NCAPH & NM_015341 & $3,03 \mathrm{E}-05$ & $-1,521$ \\
\hline NFKBIL2 & NM_013432 & $2,44 \mathrm{E}-03$ & $-1,632$ & $\mathrm{EXO1}^{\mathrm{a}}$ & NM_130398 & $3,35 \mathrm{E}-03$ & $-1,521$ \\
\hline FOXD4L3 $^{\mathrm{a}}$ & NM_199135 & $3,14 \mathrm{E}-03$ & $-1,631$ & NOS1AP & NM_014697 & $7,34 \mathrm{E}-03$ & $-1,520$ \\
\hline CALML4 & NM_033429 & $6,12 \mathrm{E}-03$ & $-1,609$ & RACGAP1 & NM_013277 & $6,33 \mathrm{E}-03$ & $-1,517$ \\
\hline DSCC1 & NM_024094 & $2,26 \mathrm{E}-03$ & $-1,602$ & CLCNKA & NM_004070 & $2,68 \mathrm{E}-03$ & $-1,517$ \\
\hline PRIM1 & NM_000946 & $2,41 \mathrm{E}-05$ & $-1,593$ & FAM133B & NM_001040057 & $7,16 \mathrm{E}-03$ & $-1,515$ \\
\hline $\mathrm{DTL}^{\mathrm{a}}$ & NM_016448 & $2,55 \mathrm{E}-04$ & $-1,591$ & $\mathrm{DUX}_{4} 4^{\mathrm{a}}$ & NM_001177376 & $8,97 \mathrm{E}-03$ & $-1,514$ \\
\hline WDHD1 & NM_007086 & $5,97 \mathrm{E}-04$ & $-1,590$ & GABRA6 & NM_000811 & $3,58 \mathrm{E}-03$ & $-1,513$ \\
\hline SUN2 & NM_015374 & 2,49E-03 & $-1,586$ & L2HGDH & NM_024884 & $3,44 \mathrm{E}-03$ & $-1,512$ \\
\hline PHF10 ${ }^{\mathrm{a}}$ & NM_018288 & $2,15 \mathrm{E}-03$ & $-1,583$ & CDKN2C & NM_001262 & $1,50 \mathrm{E}-03$ & $-1,511$ \\
\hline SKA1 & NM_001039535 & 4,42E-04 & $-1,576$ & ARHGAP19 & NM_032900 & $1,78 \mathrm{E}-03$ & $-1,510$ \\
\hline CNTNAP3 $^{a}$ & NM_033655 & $2,76 \mathrm{E}-04$ & $-1,576$ & SLFN11 & NM_001104587 & $5,13 \mathrm{E}-03$ & $-1,508$ \\
\hline RAD5 $^{\mathrm{a}}$ & NM_002875 & $2,80 \mathrm{E}-03$ & $-1,575$ & C14orf80 & NM_001134875 & $1,09 \mathrm{E}-03$ & $-1,506$ \\
\hline CDCA 8 & NM_018101 & 4,13E-04 & $-1,573$ & NCAPG & NM_022346 & $3,17 \mathrm{E}-03$ & $-1,501$ \\
\hline
\end{tabular}

The genes containing a potential binding motif for E2F1 or NF- $\mathrm{kB}$ are respectively marked by 'a' and ' $\mathrm{b}$ '. The score of all marked genes was calculated by Pscan to be higher than the average matching score for all the promoters of the genome.

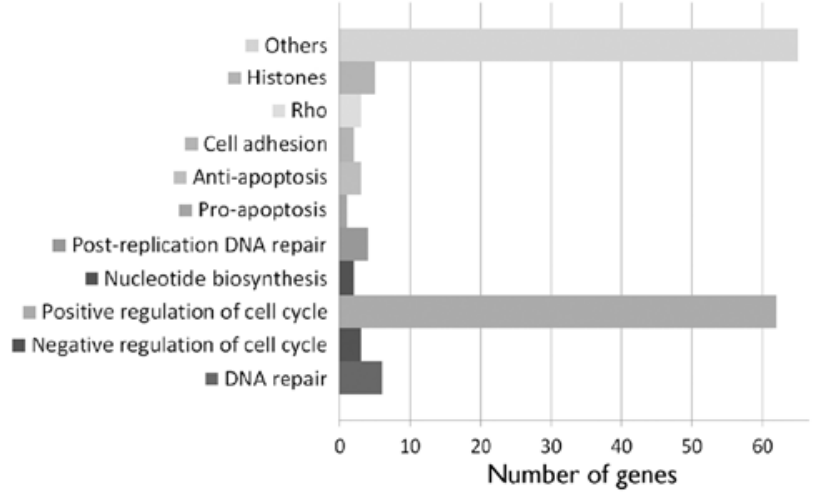

Figure 3. Number of downregulated genes in specific cellular processes $24 \mathrm{~h}$ after nickel ion irradiation (5 Gy). Almost half of the genes are involved in the positive regulation of the cell cycle. The referred processes were determined by search on the National Center for Biotechnology Information (NCBI) database.
Enrichment of transcription factor binding motifs. In order to identify the transcription factors potentially responsible for the differential gene expression upon irradiation, we scanned sequences close to the transcription start sites of these genes using Pscan (22). We found motifs for E2F1 among the transcription factor binding motifs enriched in the downregulated gene list, (p-value <10-19). On the other hand, we found two members of the REL family (RelA and NF- $\kappa$ B) with significantly enriched binding motifs in the list of upregulated genes ( $\mathrm{p}$-values $<0.05$ ).

\section{Discussion}

DNA damage persists 24 h after irradiation. We measured a significant increase in the number of $\gamma-\mathrm{H} 2 \mathrm{AX}$ foci $2 \mathrm{~h}$ following nickel ion irradiation. This number was lower than the 30 spots per nucleus that we measured on average upon 
Table II. List of the downregulated genes involved in cell cycle progression $24 \mathrm{~h}$ after $5 \mathrm{~Gy}$ of nickel ion irradiation.

\begin{tabular}{|c|c|c|c|c|c|}
\hline $\begin{array}{l}\text { DNA } \\
\text { replication }\end{array}$ & $\begin{array}{l}\text { Replication } \\
\text { forks }\end{array}$ & Spindle & Kinetochore & Centromeres & $\begin{array}{c}\text { Chromosome } \\
\text { formation/stability }\end{array}$ \\
\hline PRIM1 & MCM6 $^{\mathrm{a}}$ & HAUS8 & NDC80 & PLK4 & NCAPH \\
\hline $\mathrm{CDC}^{\mathrm{a}}$ & $\mathrm{MCM}^{\mathrm{a}}$ & KIFC1 & NUF2 & MLF1IP & DDX11 \\
\hline DSCC1 & $\mathrm{MCM}^{\mathrm{a}}$ & NUSAP $1^{a}$ & SKA1 & CEP55 & PHF10 ${ }^{\mathrm{a}}$ \\
\hline ORC1 & MCM5 $^{\mathrm{a}}$ & CDCA8 & & $\mathrm{CENPH}^{\mathrm{a}}$ & NCAPG2 \\
\hline POLA $1^{\mathrm{a}}$ & $\mathrm{MCM}^{\mathrm{a}}$ & KIF20A & & CEP57L1 & NCAPG \\
\hline GINS2 $^{\mathrm{a}}$ & $\mathrm{MCM} 10^{\mathrm{a}}$ & SKA1 & & CEP78 & CDCA2 \\
\hline \multirow[t]{7}{*}{$\mathrm{CDT}^{\mathrm{a}}$} & NFKBIL2 & BUB1 & & & \\
\hline & RFC4 & $\mathrm{KIF} 2 \mathrm{C}$ & & & \\
\hline & & PRC1 & & & \\
\hline & & BUB1B & & & \\
\hline & & KIF23 & & & \\
\hline & & ESPL1 & & & \\
\hline & & KIF11 & & & \\
\hline
\end{tabular}

The genes containing a potential binding motif for $\mathrm{E} 2 \mathrm{~F} 1$ or NF- $\mathrm{kB}$ are marked by ' $\mathrm{a}$ '. The score of all marked genes was calculated by Pscan to be higher than the average matching score for all the promoters of the genome.

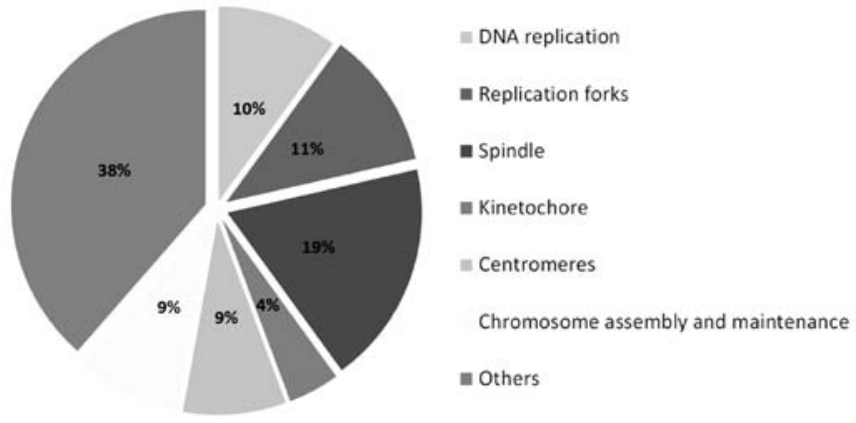

Figure 4. Pie chart representing proportions of genes downregulated $24 \mathrm{~h}$ after 5 Gy of nickel ion irradiation and involved in various processes of the cell cycle. Twenty-one percent of these genes play a role in DNA replication or in the replication forks and $41 \%$ play a role in the chromosome assembly and segregation (kinetochore, centromeres, spindle and chromosome).

X-irradiation with the same dose (data not shown). However, it is not so surprising since high LET irradiation deposits high amounts of energy along well-separated tracks. For nickel ions with a LET of $183 \mathrm{keV} / \mu \mathrm{m}$ and at a dose of $2 \mathrm{~Gy}$, we calculated an average of 6.8 direct particle hits per nucleus $\left(100 \mu \mathrm{m}^{2}\right)$, which follows a Poisson distribution. However, we observed an average of 15 spots per nucleus. This may be due to the secondary radiation from the ion track and the basal level of endogenous $\gamma-\mathrm{H} 2 \mathrm{AX}$ foci as observed in the controls.

Considering that the imaging of $\gamma-\mathrm{H} 2 \mathrm{AX}$ foci was performed at the same angle as ion tracks produced by the irradiation beam, the complexity of the damage along these tracks could not be taken into account. However, the DNA damage complexity is known to be important in high LET irradiation (23-26). Although significantly increased, the $\gamma$-H2AX spot occupancy did not seem to be able to account for the complexity of DNA damage and showed similar results to the spot number measurement. This complex DNA damage is associated with slower repair (27) and therefore leads to a more pronounced delayed cellular damage (26). Our results revealed a significant level of $\gamma-\mathrm{H} 2 \mathrm{AX}$ foci $24 \mathrm{~h}$ following nickel ion irradiation, as compared to controls; this suggests the presence of complex DNA damage.

Effects of high LET irradiation on the cell cycle. Nickel ion irradiation at a dose of $0.5 \mathrm{~Gy}$ elicited a lower gene expression response as compared to a dose of $5 \mathrm{~Gy}$, in terms of the number of regulated genes and FC. At $24 \mathrm{~h}$ post-irradiation (5 Gy), we observed an upregulation of 12 genes involved in cell cycle arrest and a downregulation of 62 genes involved in cell cycle progression, among which were 3 members of the E2F transcription factor family (E2F1, E2F2 and E2F8). Moreover, the transcription factor binding motifs for E2F1 were found to be highly enriched in the list of downregulated genes. E2F is a family of transcription factors known to control G1- to S-phase transition (28), and to regulate the expression of a large variety of genes involved in DNA replication, DNA repair and apoptosis (29). Among the E2F transcription factors, E2F1 is known to be stabilized upon DNA damage through its phosphorylation by ataxia telangiectasia-mutated (ATM) kinase, ATM and Rad3-related (ATR) kinase and checkpoint kinase 2 (CHK2), as well as through its acetylation (29). Our results suggest a major role of E2F transcription factors in the response of EA.hy926 cells to high LET irradiation.

Six components of the minichromosome maintenance (MCM) complex, a heterohexamer helicase essential for the initiation and elongation step of DNA replication (30), were downregulated. This helicase may be a target for replication checkpoints (31), and is thought to be regulated mostly through post-transcriptional modifications (32). However, our results indicate a possible transcriptional regulation of several members of the MCM complex. Apart from MCM, many of the observed downregulated genes are involved in DNA replication and in chromosome formation, maintenance and segregation, 
indicating their key role in cell cycle regulation in response to high LET radiation. Of note, we also reported the downregulation of 4 genes involved in post-replication DNA repair (UNG, UPF3A, MSH2 and MSH6), which may be silenced in the absence of active replication.

During this study, irradiation was performed on proliferating endothelial cells. The results gathered on cell cycle gene expression are therefore of moderate interest for mature blood vessels where proliferation is limited. However, as far as hadron therapy is concerned, our data indicate that high LET radiation may have a significant impact on the cellular proliferation of newly formed vascular vessels in the vicinity of the targeted tumor.

DNA damage response, oxidative stress and apoptosis. The expression of several genes involved in the DNA damage response, oxidative stress response and apoptosis was induced $24 \mathrm{~h}$ after $5 \mathrm{~Gy}$ of nickel ion irradiation, with a concomitant reduction of genes involved in DNA repair. However, these effects were not significant at a dose of $0.5 \mathrm{~Gy}$, at either time points ( 8 and $24 \mathrm{~h}$ ). These results suggest that a high dose of nickel ion irradiation induces a global DNA damage response, accompanied by cell cycle arrest and an increase in proapoptotic gene expression $24 \mathrm{~h}$ after irradiation.

Impact of radiation on genes related to cell adhesion. The impermeability of the endothelium is essential for the vasculature integrity and is determined by the cooperation of cell junctions and the cytoskeleton $(33,34)$. In turn, adhesion molecules regulate cell homeostasis, growth and apoptosis (33). A number of cellular pathways are known to regulate cell adhesion in endothelial cells. These include growth factors, Rho GTPases, protein kinases and calcium signaling $(34,35)$. The alteration of these pathways or of adhesion molecules may trigger the radiation-induced retraction observed by others in endothelial cells $(13,14)$. Our study identified the differential expression of a number of genes known to be involved in cell adhesion (CEACAM1 and NEU1), cytoskeleton architecture (TUBA4A, LIMA1 and PLS1), Rho signaling (ARHGAP19, ARHGAP11B and RACGAP1) and calcium metabolism (ORAI3, CAMK2N1 and CALML4) $24 \mathrm{~h}$ after 5 Gy of nickel ion irradiation, which are potentially involved in endothelial cell retraction.

Expression of cytokines and chemokines. Inflammatory responses mediated by endothelial cells are believed to be involved in radiation-induced cardiovascular disease (7). Our study revealed the upregulation of 8 cytokines or chemokines that may be linked to inflammation (CXCL5, TGFA, TRIM22, TNFSF9, EBI3, IL-6, IL-11 and CD70). Of note, a search for transcription factor binding motifs that are significantly enriched in the list of upregulated genes upon 5 Gy of irradiation, revealed 2 members of the REL family (RelA and NF- $\kappa$ B). This family of transcription factors induces the expression of a multitude of genes, such as cytokines, proliferation, prosurvival and anti-apoptotic genes (36). For instance, we found IL-6 to be upregulated after 5 Gy of nickel ion irradiation. IL-6 expression was also shown to be upregulated by lowdose radiation therapy (10). IL-6 is known to be activated by $\mathrm{NF}-\kappa \mathrm{B}(36,37)$ and is thought to play a role in radiation-induced cardiovascular disease $(1,7)$. The secretion of cytokines may also affect non-irradiated cells by a bystander effect. Indeed, in human fibroblasts, the external addition of IL- 6 has been shown to increase $\gamma \mathrm{H} 2 \mathrm{AX}$ spot occupancy (38). The activation of $\mathrm{NF}-\kappa \mathrm{B}$ may be linked to the transcription factor, signal transducer and activator of transcription 3 (STAT3) (37), of which we also found significant binding motif enrichment.

In conclusion, we observed a downregulation of multiple genes involved in cell division, particularly at $24 \mathrm{~h}$ after nickel ion irradiation. Our results suggest an important role for E2F transcription factors in this process. The endothelial function being based on a plethora of intercellular interactions within a dynamic structure involving cell movements and turnover, cell cycle arrest may play a role in the radiation-induced cardiovascular disease. On the other hand, we observed an upregulation of various cytokines which may be induced by NF- $\kappa \mathrm{B}$. Other studies have also suggested that these cytokines may be linked to radiation-induced cardiovascular disease (10). The effects on gene expression were observed upon high doses of acute irradiation and are less relevant to space exploration. However, during hadron therapy, healthy tissues surrounding tumors, such as endothelial cells, may be subjected to high doses, which may lead to complications. In this study, we identified a multitude of potential molecular targets for further mechanistic studies out of which the gene expression changes upon high doses of nickel ion irradiation may be important for patients treated with hadron-therapy.

\section{Acknowledgements}

This study was supported by 4 PRODEX/BELSPO/ESA contracts (C90-303, C90-380, C90-391 and 42-000-90-380) and the ESA IBER-2 program. The authors wish to thank Professor Marco Durante for providing access to the GSI irradiation facilities.

\section{References}

1. Schultz-Hector S and Trott KR: Radiation-induced cardiovascular diseases: is the epidemiologic evidence compatible with the radiobiologic data? Int J Radiat Oncol Biol Phys 67: 10-18, 2007.

2. Blaber E, Marçal H and Burns BP: Bioastronautics: the influence of microgravity on astronaut health. Astrobiology 10: 463-473, 2010.

3. Brinckmann E (ed): Biology in Space and Life on Earth: Effects of Spaceflight on Biological Systems. WILEY-VCH Verlag GmbH \& Co. KGaA, Weinheim, 2007.

4. Rong Y and Welsh J: Basics of particle therapy II biologic and dosimetric aspects of clinical hadron therapy. Am J Clin Oncol 33: 646-649, 2010.

5. Blakely EA and Chang PY: Biology of charged particles. Cancer J 15: 271-284, 2009.

6. Halle M, Hall P and Tornvall P: Cardiovascular disease associated with radiotherapy: Activation of nuclear factor kappa-B. J Intern Med 269: 469-477, 2011.

7. Hildebrandt G: Non-cancer diseases and non-targeted effects. Mutat Res 687: 73-77, 2010.

8. Rödel F, Frey B, Capalbo G, et al: Discontinuous induction of $\mathrm{x}$-linked inhibitor of apoptosis in ea.Hy.926 endothelial cells is linked to NF- $\kappa \mathrm{B}$ activation and mediates the anti-inflammatory properties of low-dose ionising-radiation. Radiother Oncol 97: 346-351, 2011.

9. Rödel F, Hofmann D, Auer J, et al: The anti-inflammatory effect of low-dose radiation therapy involves a diminished CCL20 chemokine expression and granulocyte/endothelial cell adhesion. Strahlenther Onkol 184: 41-47, 2008. 
10. Rödel F, Keilholz L, Herrmann M, Sauer R and Hildebrandt G: Radiobiological mechanisms in inflammatory diseases of low-dose radiation therapy. Int J Radiat Biol 83: 357-366, 2007.

11. Rödel F, Schaller U, Schultze-Mosgau S, et al: The induction of TGF-beta(1) and NF-kappaB parallels a biphasic time course of leukocyte/endothelial cell adhesion following low-dose X-irradiation. Strahlenther Onkol 180: 194-200, 2004.

12. Ando K, Ishibashi $\mathrm{T}$, Ohkawara $\mathrm{H}$, et al: Crucial role of membrane type 1 matrix metalloproteinase (MT1-MMP) in Rhoa/Rac1-dependent signaling pathways in thrombin-stimulated endothelial cells. J Atheroscler Thromb 18: 762-773, 2011

13. Kantak SS, Diglio CA and Onoda JM: Low dose radiation-induced endothelial cell retraction. Int J Radiat Biol 64: 319-328, 1993.

14. Onoda JM, Kantak SS and Diglio CA: Radiation induced endothelial cell retraction in vitro: correlation with acute pulmonary edema. Pathol Oncol Res 5: 49-55, 1999.

15. Gabrys D, Greco O, Patel G, Prise KM, Tozer GM and Kanthou C: Radiation effects on the cytoskeleton of endothelial cells and endothelial monolayer permeability. Int J Radiat Oncol Biol Phys 69: 1553-1562, 2007.

16. Pluder F, Barjaktarovic Z, Azimzadeh O, et al: Low-dose irradiation causes rapid alterations to the proteome of the human endothelial cell line EA.hy926. Radiat Environ Biophys 50: 155-166, 2011.

17. Grabham P, Hu B, Sharma P and Geard C: Effects of ionizing radiation on three-dimensional human vessel models: differential effects according to radiation quality and cellular development. Radiat Res 175: 21-28, 2011.

18. Takahashi Y, Teshima T, Kawaguchi N, Hamada Y, Mori S, Madachi A, Ikeda S, Mizuno H, Ogata T, Nojima K, Furusawa Y and Matsuura N: Heavy ion irradiation inhibits in vitro angiogenesis even at sublethal dose. Cancer Res 63: 4253-4257, 2003.

19. Kiyohara H, Ishizaki Y, Suzuki Y, Katoh H, Hamada N, Ohno T, Takahashi T, Kobayashi Y and Nakano T: Radiation-induced ICAM- 1 expression via TGF- $\beta 1$ pathway on human umbilical vein endothelial cells; comparison between X-ray and carbon-ion beam irradiation. J Radiat Res 52: 287-292, 2011.

20. Haberer T, Becher W, Schardt D and Kraft G: Magnetic scanning system for heavy ion therapy. Nucl Instrum Methods Phys Res A 330: 296-305, 1993.

21. De Vos WH, Van Neste L, Dieriks B, Joss GH and Van Oostveldt P: High content image cytometry in the context of subnuclear organization. Cytometry A 77: 64-75, 2010.

22. Zambelli F, Pesole G and Pavesi G: Pscan: finding over-represented transcription factor binding site motifs in sequences from co-regulated or co-expressed genes. Nucleic Acids Res 37: W247-W252, 2009.
23. Fokas E, Kraft G, An H and Engenhart-Cabillic R: Ion beam radiobiology and cancer: time to update ourselves. Biochim Biophys Acta 1796: 216-229, 2009.

24. Held KD: Effects of low fluences of radiations found in space on cellular systems. Int J Radiat Biol 85: 379-390, 2009.

25. Costes SV, Boissière A, Ravani S, Romano R, Parvin B and Barcellos-Hoff MH: Imaging features that discriminate between foci induced by high- and low-let radiation in human fibroblasts. Radiat Res 165: 505-515, 2006.

26. Blakely EA and Kronenberg A: Heavy-ion radiobiology: new approaches to delineate mechanisms underlying enhanced biological effectiveness. Radiat Res 150: S126-S145, 1998.

27. Chappell LJ, Whalen MK, Gurai S, Ponomarev A, Cucinotta FA and Pluth JM: Analysis of flow cytometry DNA damage response protein activation kinetics after exposure to $\mathrm{x}$ rays and high-energy iron nuclei. Radiat Res 174: 691-702, 2010.

28. Dyson N: The regulation of E2F by pRB-family proteins. Genes Dev 12: 2245-2262, 1998

29. Biswas AK and Johnson DG: Transcriptional and nontranscriptional functions of E2F1 in response to DNA damage. Cancer Res 72: 13-17, 2012.

30. Costa A and Onesti S: The MCM complex: (just) a replicative helicase? Biochem Soc Trans 36: 136-140, 2008.

31. Forsburg SL: The MCM helicase: Linking checkpoints to the replication fork. Biochem Soc Trans 36: 114-119, 2008.

32. Chuang CH, Yang D, Bai G, Freeland A, Pruitt SC and Schimenti JC: Post-transcriptional homeostasis and regulation of MCM2-7 in mammalian cells. Nucleic Acids Res 40: 4914-4924, 2012.

33. Dejana E, Orsenigo F, Molendini C, Baluk P and McDonald DM: Organization and signaling of endothelial cell-to-cell junctions in various regions of the blood and lymphatic vascular trees. Cell Tissue Res 335: 17-25, 2009.

34. Prasain $\mathrm{N}$ and Stevens T: The actin cytoskeleton in endothelial cell phenotypes. Microvasc Res 77: 53-63, 2009.

35. Bogatcheva NV and Verin AD: The role of cytoskeleton in the regulation of vascular endothelial barrier function. Microvasc Res 76: 202-207, 2008.

36. Oeckinghaus A and Ghosh S: The NF-kappaB family of transcription factors and its regulation. Cold Spring Harb Perspect Biol 1: a000034, 2009.

37. Karin M: NF-kappaB as a critical link between inflammation and cancer. Cold Spring Harb Perspect Biol 1: a000141, 2009.

38. Dieriks B, De Vos WH, Derradji H, Baatout $S$ and Van Oostveldt P: Medium-mediated DNA repair response after ionizing radiation is correlated with the increase of specific cytokines in human fibroblasts. Mutat Res 687: 40-48, 2010. 